\title{
$P$-Wave Positron-Hydrogen Scattering, Annihilation, and Positronium Formation
}

\author{
Anand K. Bhatia \\ Heliophysics Science Division, NASA/Goddard Space Flight Center, Greenbelt, MD 20771, USA; \\ Anand.K.Bhatia@nasa.gov \\ Academic Editor: James Babb \\ Received: 10 January 2017; Accepted: 7 April 2017; Published: 18 April 2017
}

\begin{abstract}
In a previous paper (Bhatia A.K. 2016), a hybrid theory for the scattering of positrons from hydrogen atoms was applied to calculate $S$-wave phase shifts, annihilation, and positronium formation cross sections. This approach is now being applied to calculate $P$-wave positron-hydrogen scattering. The present results, obtained using short-range correlation functions along with long-range correlations in the Schrödinger equation at the same time, agree very well with the results obtained in an earlier calculation by Bhatia et al. (1974), using the Feshbach projection operator formalism. In these earlier calculations, the correction due to the long-range correlations was applied to the variational results. In spite of the fact that this ad hoc correction destroyed the variational bound, the final results have been considered accurate. Annihilation cross-sections, positronium formation cross-sections, calculated in the distorted-wave approximation, are also presented.
\end{abstract}

Keywords: positron scattering; annihilation; positronium formation

\section{Introduction}

Various approximations have been used to study scattering of electrons and positrons by hydrogenic systems. The distortion produced in the target by the incident particle has been taken into account in the ansatz for the wave function for the scattering process by the method of polarized orbitals of Temkin (1959) [1]. However, this method is not variational and therefore provides no bounds on the calculated phase shifts. The alternate method is to introduce separate short-range and long-range correlation functions and amalgamate them into a scattering problem via an optical potential in the scattering equation, thereby replacing the many-body Schrödinger equation with a single-particle Schrödinger equation. This approach was carried out in the formalism of the hybrid theory of Bhatia $(2007,2008,2016)$ [2-4]. The phase shifts obtained are rigorous lower bounds to the exact phase shifts. We now apply this approach to the $P$-wave scattering of positrons by hydrogen atoms. The details of this formalism have been given in previous publications of Bhatia $(2007,2008)[2,3]$ and therefore, we only briefly describe the method. The wave function is given by

$$
\Psi_{L}\left(\vec{r}_{1}, \vec{r}_{2}\right)=\frac{u_{L}\left(r_{1}\right)}{r_{1}} Y_{L 0}\left(\Omega_{1}\right) \Phi^{p o l}\left(r_{1}, r_{2}\right)+\sum_{\lambda=1}^{N} C_{\lambda} \Phi_{L}^{\lambda}\left(\vec{r}_{1}, \vec{r}_{2}\right),
$$

Since positrons and electrons are distinguishable particles, the Pauli principle need not be invoked. However, positronium formation and annihilation of a positron with the bound electron can take place during the scattering. The positron coordinate is given by $\vec{r}_{1}$; the electron coordinate by $\vec{r}_{2}$; and the summation over $\lambda$ is from 1 to $N$, the number of terms in the expansion; $C_{\lambda}$ are the unknown coefficients; and $\Phi_{L}^{\lambda}$ is the correlation function for the angular momentum $L$. The correlation functions 
are short-range in $\vec{r}_{1}$ and $\vec{r}_{2}$, and are normalized to unity. The expectation values of these correlation functions are given by

$$
<\Phi_{L}^{\lambda}|H| \Phi_{L}^{\lambda}>=\varepsilon(\lambda)
$$

We use Rydberg units throughout $\left[e^{2}=2, \hbar=1, m_{e}=0.5\right.$, and $\left.a_{0}=1\right]$. In Equation (2), $H$ is the Hamiltonian and $\varepsilon(\lambda)$ is the expectation value of $H$. We have $H$ in these units:

$$
H=-\nabla_{1}^{2}-\nabla_{2}^{2}+\frac{2 Z}{r_{1}}-\frac{2 Z}{r_{2}}-\frac{2}{r_{12}} .
$$

$\mathrm{Z}$ is the nuclear charge in the above equation. In order to include polarization of the target, the effective target function can be written as:

$$
\Phi^{p o l}\left(\vec{r}_{1}, \vec{r}_{2}\right)=\varphi_{0}\left(\vec{r}_{2}\right)+\frac{\chi_{\beta}\left(r_{1}\right)}{r_{1}^{2}} \frac{u_{1 s \rightarrow p}\left(r_{2}\right)}{r_{2}} \frac{\cos \left(\theta_{12}\right)}{(Z \pi)^{0.5}}
$$

The function $u_{1 s \rightarrow p}$ is given by:

$$
u_{1 s \rightarrow p}\left(r_{2}\right)=e^{-Z r_{2}}\left(\frac{Z}{2} r_{2}^{3}+r_{2}^{2}\right) .
$$

The target function is given by:

$$
\varphi_{0}\left(r_{2}\right)=\sqrt{\frac{Z^{3}}{\pi}} e^{-Z r_{2}}
$$

In Equation (4), $\chi_{\beta}\left(r_{1}\right)$ is the smooth cutoff function which guarantees that $\chi_{\beta}\left(r_{1}\right) / r_{1}^{2} \rightarrow 0$ for $r_{1} \rightarrow 0$. This function is given by

$$
\chi_{\beta}\left(r_{1}\right)=\left(1-e^{-\beta r_{1}}\right)^{n}
$$

where $n \geq 3$. This function ensures that the polarization takes place throughout the range, rather than only for $r_{1}>r_{2}$ as in [1]. Furthermore, the function gives us another nonlinear parameter $\beta$ which is a function of $k$, the incident positron momentum, along with the exponent $n$. The cutoff function $\chi_{S T}$ used in the $S$-wave calculation [4] could have been used here too. However, the cutoff function given in Equation (7) allows flexibility to optimize the phase shifts. The nonlinear parameter $\beta$ and the exponent $n$ can be varied. Therefore, the cutoff function is different for different $k$ which is not true of the cutoff function $\chi_{S T}$. The angle $\theta_{12}$ is the angle between $\vec{r}_{1}$ and $\vec{r}_{2}$. In Equation (1), $L$ is the angular momentum; $\mathrm{u}_{L}$ is the scattering function; and the function $\Phi_{L}$ is the correlation function which can be written in terms of the generalized "radial" functions, which depend upon the radial coordinates and the Euler angles introduced by Bhatia and Temkin (1964) [5]:

$$
\Phi_{L}=\sum_{\kappa}\left[f_{L}^{\kappa,+1}\left(r_{1}, r_{2}, r_{12}\right) D_{L}^{\kappa,+1}(\theta, \varphi, \psi)+f_{L}^{\kappa,-1}\left(r_{1}, r_{2}, r_{12}\right) D_{L}^{\kappa,-1}(\theta, \varphi, \psi)\right]
$$

In the above equation, $D^{\kappa, \varepsilon}(\varepsilon=+o r-)$ are the modified spherical harmonics which depend on the Euler angles $\theta, \varphi$, and $\psi$. Here, $\kappa$ is equal to 1 and the radial functions are given by:

$$
\begin{aligned}
& f_{L}^{\kappa,+1}\left(r_{1}, r_{2}, r_{12}\right)=\cos \left(\theta_{12} / 2\right) \sum_{l m n}\left[C_{l m n} R_{l m n}\left(r_{1}, r_{2}, r_{12} ; \gamma, \delta\right)+D_{l m n} R_{l m n}\left(r_{2}, r_{1}, r_{12} ; \gamma, \delta\right)\right] \\
& f_{L}^{\kappa,-1}\left(r_{1}, r_{2}, r_{12}\right)=\sin \left(\theta_{12} / 2\right) \sum_{l m n}\left[C_{l m n} R_{l m n}\left(r_{1}, r_{2}, r_{12} ; \gamma, \delta\right)-D_{l m n} R_{l m n}\left(r_{2,}, r_{1}, r_{12} ; \gamma, \delta\right)\right]
\end{aligned}
$$

In Equations (9) and (10), $C_{l m n}$ and $D_{l m n}$ are independent eigenvectors because the target is in an s-state when the incident positron is in a $P$-wave, giving the total angular momentum equal to 1 ; while the target can be in a $p$-state when the incident positron is in an s-state, giving the total angular 
momentum equal to 1 . Both states must be considered for the $P$-wave scattering. The second terms in Equations (9) and (10) can have different nonlinear parameters than the first term. However, we find that the nonlinear parameters $\gamma, \delta$, and $\beta$ are sufficient to obtain converged phase shifts. The radial function in the above equations is given by:

$$
R_{l m n}\left(r_{1}, r_{2}, r_{12} ; \gamma, \delta\right)=e^{-\gamma r_{1}-\delta r_{2}} r_{1}^{l} r_{2}^{m} r_{12}^{n}
$$

where $l \geq 1, m \geq 0$, and $n \geq 0$. The sum $l+m+n$ is equal to $\omega=1,2,3,4,5$, and 6 as given in Pekeris (1958) [6]. These values correspond to $4,10,20,35,56$, and 84 correlation terms in each symmetry. The variation with respect to $C_{\lambda}$, as indicated by Bhatia (2007) [2], in the functional

$$
I=\left\langle\Psi_{L}\left(\vec{r}_{1}, \vec{r}_{2}\right)|H-E| \Psi_{L}\left(\vec{r}_{1}, \vec{r}_{2}\right)\right\rangle
$$

gives:

$$
C_{\lambda}=\frac{\left\langle V_{\lambda}\left(r_{1}\right) u\left(r_{1}\right)\right\rangle}{E-\varepsilon(\lambda)}
$$

In the above equation $E=k^{2}-Z^{2}$, we get the equation for the scattering function $u(r)$ :

$$
\left[D(r) \frac{d^{2}}{d r^{2}}-\frac{2}{r^{2}}+k^{2}+V_{d}+V_{p o l}-V_{o p t}^{p o l}\right] u(r)=0
$$

We give the various quantities:

$$
D(r)=1+\frac{43}{8 Z^{6}}\left(\frac{\chi_{\beta}(r)}{r^{2}}\right)^{2}
$$

The direct potential is given by:

$$
V_{d}(r)=-\frac{2(Z-1)}{r}-2 e^{-2 Z r}\left(Z+\frac{1}{r}\right)
$$

and

$$
V_{p o l}(r)=\left(x_{1}+x_{3}\right)+x_{2} \frac{d}{d r}
$$

The quantities in the above equation are

$$
\begin{gathered}
x_{1}=2 \chi_{\beta}(r) \alpha(r) /(\mathrm{Zr})^{4} \\
x_{2}=B_{1}(r) \frac{43}{4 Z^{6}} \frac{\chi_{\beta}(r)}{r^{2}} \\
x_{3}=-4.5\left(\frac{\chi_{\beta}(r)}{(Z r)^{2}}\right)^{2}+\frac{43}{8 Z^{6}}\left(B_{2}(r)+y 3\right) \frac{\chi_{\beta}(r)}{r^{2}}+\frac{d(r)}{Z}\left(\frac{\chi_{\beta}(r)}{(Z r)^{2}}\right)^{2} \\
y 3=\left(-\frac{2 Z}{r}-\frac{4}{r^{2}}+k^{2}\right) \frac{\chi_{\beta}(r)}{r^{2}} \\
B_{1}(r)=n \beta e^{-\beta r}\left(1-e^{-\beta r}\right)^{n-1} / r^{2}-2 \chi_{\beta}(r) / r^{3} \\
B_{2}(r)=n \beta e^{-\beta r}(y 4) / r^{2}+2\left(\chi_{\beta}(r) / r^{4}-B_{1}(r) / r\right) \\
y 4=-(\beta+2 / r)\left(1-e^{-\beta r}\right)^{n-1}+\beta e^{-\beta r}(n-1)(n-2)\left(1-e^{-\beta r}\right)^{n-2} \\
d(r)=\frac{43}{4 Z r}+\frac{48}{(Z r)^{3}}-e^{-2 Z r}\left(\frac{(Z r)^{4}}{2}+4.5(Z r)^{3}+18(Z r)^{2}+45 Z r+81.25+\frac{106.75}{Z r}+\frac{96}{(Z r)^{2}}+\frac{48}{(Z r)^{3}}\right)
\end{gathered}
$$


The polarizabilty of the target is obtained from the following expression as $r \rightarrow \infty$ :

$$
\alpha(r) / Z^{4}
$$

where

$$
\alpha(r)=4.5-e^{-2 Z r}\left((Z r)^{4}+5(Z r)^{3}+9(Z r)^{2}+9(Z r)+4.5\right)
$$

The optical potential is given by:

$$
V_{o p}^{p o l}(r) u(r)=r \sum_{s} \frac{\left\langle Y_{10}\left(\Omega_{1}\right) \Phi^{p o l}\left(\vec{r}_{1}, \vec{r}_{2}\right)|H-E| \Phi_{0}^{s}\right\rangle\left\langle\Phi_{0}^{s}|H-E| \Psi_{0}^{\prime}\right\rangle}{E-\varepsilon(s)}
$$

Here, $\Psi_{0}^{\prime}$ is the wave function given in Equation (1) without the correlation term. The phase shifts are inferred from the scattering function $u_{L} \equiv u$ for $r$ tending to infinity:

$$
u(r)=\frac{\sin (k r-L \pi / 2+\eta)}{k r}
$$

In Table 1 , we show the convergence of phase shifts $\eta$ (radians) for $k=0.4$ with the number of terms in the correlation function given in Equation (1). The total number of terms is $2 N$ because there are two independent eigenvectors $C_{l m n}$ and $D_{l m n}$. The nonlinear parameters $\gamma, \delta$, and $\beta$ are also given, while keeping the exponent $n$ fixed at 3 . By $N=56$, the phase shift has converged to three significant figures. However, calculations have been carried out to $N=84$ for all values of $k$, the incident momentum, in order to have converged results and to use the resulting wave functions for the calculation of $Z_{e f f}$. In Table 2, results, which have not been extrapolated, are given for $k=0.1$ to 0.7 , the exponent $n=3$ for all values of $k$. For $k=0.3$, phase shift for $2 N=168$ is not given because there is a loss of accuracy in the numerical calculation: Instead of increasing with the number of terms, the phase shift decreases to 0.065236 . It is seen that the present results are higher than those of Bhatia et al. (1974) [7], obtained without extrapolation. As indicated above, the present results include short-range and long-range correlations at the same time and have variational lower bounds to the exact phase shifts.

Table 1. Convergence of phase shifts $\eta$ (radians) for $\mathrm{e}^{+}-\mathrm{H}$ scattering with respect to the number of terms $N$ for $k=0.4$.

\begin{tabular}{cccccc}
\hline $\boldsymbol{N}$ & $\mathbf{2 N}$ & $\gamma$ & $\boldsymbol{\delta}$ & $\boldsymbol{\beta}$ & $\boldsymbol{\eta}$ \\
\hline 4 & 8 & 0.74 & 0.90 & 0.366 & 0.06889 \\
10 & 20 & 0.67 & 0.75 & 0.275 & 0.09279 \\
20 & 40 & 0.75 & 0.72 & 0.277 & 0.09904 \\
35 & 70 & 0.75 & 0.68 & 0.277 & 0.10019 \\
56 & 112 & 0.75 & 0.68 & 0.277 & 0.10031 \\
84 & 168 & 0.75 & 0.68 & 0.277 & 0.10047 \\
\hline
\end{tabular}

Over the years, reviews on interactions of positrons with atoms and ions have been published. Among them are reviews by Drachman (1971) [8], Ghosh et al. (1982) [9], Bhatia (2014) [10], and Kadyrov and Bray (2016) [11]. 
Table 2. Phase shifts $\eta$ (radians) for $\mathrm{e}^{+}-\mathrm{H}$ scattering for $2 N=168$ for various $k$ and comparison with previous calculations.

\begin{tabular}{cccc}
\hline $\boldsymbol{K}$ & Present & A & B \\
\hline 0.1 & 0.008871 & 0.00876 & 0.008 \\
0.2 & 0.032778 & 0.03251 & 0.032 \\
0.3 & $0.065964^{\mathrm{a}}$ & 0.065556 & 0.064 \\
0.4 & 0.10047 & 0.10005 & 0.099 \\
0.5 & 0.13064 & 0.13027 & 0.130 \\
0.6 & 0.15458 & 0.15410 & 0.153 \\
0.7 & 0.17806 & 0.17742 & 0.175 \\
\hline
\end{tabular}

A: Bhatia et al. (1974) [7]; B: Armstead (1968) [12]. ${ }^{\text {a }} k=0.3$ results are for $2 N=112$.

\section{2. $Z_{\text {eff }}$}

In addition to the scattering, there is a possibility of annihilation of an incoming positron and an atomic electron with the emission of two gamma rays as given by Ferrell (1956) [13]:

$$
\sigma_{a}\left(\pi a_{0}^{2}\right)=Z_{e f f} \alpha^{3} / k
$$

where $\alpha=e^{2} / \hbar c$ is the fine-structure constant and $a_{0}$ is the Bohr radius. Positronium annihilation has been observed in solar flares by Crannell et al. (1976) [14], from the center of our galaxy by Leventhal et al. (1978) [15], and from gamma ray bursts by Cline (1978) [16]. The quantity $Z_{\text {eff, which measures }}$ the overlap of the target electron with the positron, as indicated by DiRienzi and Drachman (2003) [17], approaches $Z$, the number of electrons for a free positron. For hydrogen:

$$
Z_{e f f}=\iint d \vec{r}_{1} d \vec{r}_{2}\left|\Psi_{L}\left(\vec{r}_{1}, \vec{r}_{2}\right)\right|^{2} \delta\left(\vec{r}_{1}-\vec{r}_{2}\right),
$$

The normalization of $u_{L}\left(r_{1}\right)$ in the above $\Psi\left(\vec{r}_{1}, \vec{r}_{2}\right)$ for $r_{1} \rightarrow \infty$ is a plane-wave normalization:

$$
u_{L}=[4 \pi(2 L+1)]^{1 / 2} k^{-1} \sin \left(k r_{1}-\frac{1}{2} \pi L+\eta\right)
$$

For angular momentum $L$, this is equivalent to

$$
\exp \left(i k . r_{1}\right)=i^{L}[4 \pi(2 L+1)]^{\frac{1}{2}} j_{L}\left(k r_{1}\right) Y_{L 0}(\theta)
$$

In Table 3, we present values for $Z_{\text {eff }}$ for $L=1$ as a function of $k$ below the positronium formation threshold. The present results are compared with the previous ones of Bhatia et al (1977) [18] and also with those obtained by Humberston (1977) [19]. The agreement is quite good.

Table 3. $Z_{\text {eff }}(1)$ as a function of the incident positron momentum $k$, obtained in this calculation and compared with previous calculations. The results from this publication are for $2 N=168$, except for $k=0.3$.

\begin{tabular}{cccccc}
\hline $\boldsymbol{k} \backslash \mathbf{2 N}$ & $\mathbf{7 0}$ & $\mathbf{1 1 2}$ & $\mathbf{1 6 8}$ & $\mathbf{A}$ & $\mathbf{B}$ \\
\hline 0.1 & 0.122006 & 0.130993 & 0.136785 & 0.1338 & 0.1335 \\
0.2 & 0.528257 & 0.523821 & 0.540436 & 0.53994 & 0.5366 \\
0.3 & 1.054067 & 1.11009 & $\ldots \ldots$ & 1.1027 & 1.114 \\
0.4 & 1.65737 & 1.71498 & 1.74840 & 1.7692 & 1.719 \\
0.5 & 2.21526 & 2.26710 & 2.32396 & 2.3391 & 2.353 \\
0.6 & 2.71414 & 2.76818 & 2.81990 & 2.84988 & 2.823 \\
0.7 & 3.28940 & 3.49006 & 3.62483 & 3.67030 & 3.637 \\
\hline
\end{tabular}

A: Bhatia et al (1977) [18], B: Humberston (1977), quoted in Bhatia et al. (1977) [18]. 


\section{Positronium Formation}

Formation of positronium, the bound state of an electron and a positron, takes place when the incident positron captures an electron of the hydrogen atom before the positron and electron annihilate each other:

$$
e^{+}+H(1 s) \rightarrow P s+P
$$

where $P_{S}$ is the positronium atom and $P$ is the proton. Using the distorted-wave approximation, Khan and Ghosh (1983) [20] have carried out calculations using the method of polarized orbitals of Temkin (1959) [1]. The present calculations are similar to those of Khan and Ghosh. However, the scattering wave function is calculated variationally, Bhatia (2007) [2]. The details of the calculation for S-wave have been given in Bhatia (2016) [4] which can be easily generalized to higher partial waves, and are not repeated here. Similarly, various experiments carried out on the formation of positronium are given in Bhatia (2016) [4] and are not repeated here. It should be pointed out that the continuum function has a plane-wave normalization as in Equation (33). We have used $\mu_{i} \mu_{f}=1 / 2$ in Rydberg units. Cross-sections obtained without and with polarization are given in Table 4 and compared with those obtained by Khan and Ghosh (1983) [20]. The present cross-sections have a maximum at $k^{2}=0.75$. The cross-sections increase when the polarization of the target is taken into account. It is seen that the present results are lower than those of Khan and Ghosh (1983) [20]. It could be due to the fact that they have incorrect sign [negative sign instead of positive sign] in Equation (4), as explained in Bhatia (2016) [4]. The present calculations and those of Khan and Ghosh do not include correlation terms.

The integrals occurring in the positronium formation calculation have been carried out using the Fourier transforms, as indicated in [4]. The correlation terms have high powers of $r_{2}$ and $r_{12}$ and it becomes very difficult to calculate all the Fourier transforms that are required.

Table 4. Comparison of the present results for positronium formation cross-section $\left(\pi a_{0}^{2}\right)$ in the partial wave $L=1$ with those obtained in other calculations.

\begin{tabular}{ccccc}
\hline $\boldsymbol{k}_{\boldsymbol{i}}^{\mathbf{2}}$ & $\mathbf{A}$ & $\mathbf{B}$ & $\mathbf{C}$ & $\mathbf{D}$ \\
\hline 0.5041 & 0.004598 & 0.004766 & 0.018574 & 0.013922 \\
0.5476 & 0.12429 & 0.12930 & & \\
0.5625 & 0.16505 & 0.17195 & 0.59291 & 0.438436 \\
0.5929 & 0.23453 & 0.24500 & & \\
0.64 & 0.30502 & 0.32006 & 1.07621 & 0.783038 \\
0.6724 & 0.33073 & 0.34816 & & \\
0.7225 & 0.34771 & 0.36783 & 1.20910 & 0.866246 \\
0.75 & 0.34788 & 0.36902 & 1.2039 & 0.858605 \\
0.81 & 0.33529 & 0.35780 & & \\
0.9025 & 0.30000 & 0.32305 & & \\
1.00 & 0.25580 & 0.27805 & & \\
\hline
\end{tabular}

A: Present results, without polarization; B: present results with polarization; C: Khan and Ghosh (1983) [20] results without polarization; and D: their results with polarization.

\section{Conclusions}

Using the hybrid theory of scattering of Bhatia (2007) [2], we have calculated phase shifts which are lower bounds to the exact phase shifts. This calculation includes the contribution of the long-range interaction $-1 / r^{4}$ variationally and our results agree with the previous calculations of Bhatia et al. (1974) [7] and those of Armstead (1968) [12]. The scattering functions have been used to calculate $Z_{e f f}$ and positronium formation cross-sections in the distorted wave approximation. The hybrid theory has been used previously to calculate accurate phase shifts, Bhatia (2007) [2]; resonance parameters, Bhatia (2008) [3]; and photoabsorption cross sections, Bhatia (2013) [21]. It is expected that the present results are accurate to at least four decimal places. 
Acknowledgments: Thanks are extended to R. J. Drachman for helpful discussions, and for critical reading of the manuscript. Calculations were carried out in quadruple precision using the Discover computer of NASA Center for Computation Science.

Conflicts of Interest: The author declares no conflict of interest.

\section{References}

1. Temkin, A. A Note on the Scattering of Electrons from Atomic Hydrogen. Phys. Rev. 1959, 116, 358. [CrossRef]

2. Bhatia, A.K. Hybrid theory of Electron-hydrogen Elastic Scattering. Phys. Rev. A 2007, 75, 032713. [CrossRef]

3. Bhatia, A.K. Applications of the Hybrid Theory to the Scattering of Electrons from $\mathrm{He}^{+}$and $\mathrm{Li}^{2+}$ and Resonances in these Systems. Phys. Rev. A 2008, 77, 052707. [CrossRef]

4. Bhatia, A.K. Positron-hydrogen Scattering, Annihilation, and Positronium Formation. Atoms 2016, 4, 27. [CrossRef]

5. Bhatia, A.K.; Temkin, A. Symmetric Euler-Angle Decomposition of the Two-Electron Fixed-Nucleus Problem. Rev. Mod. Phys. 1964, 36, 1050. [CrossRef]

6. Pekeris, C.L. Ground State of Two-Electron Atoms. Phys. Rev. 1958, 112, 1649. [CrossRef]

7. Bhatia, A.K.; Temkin, A.; Eiserike, H. Rigorous precision $P$-wave Positron-Hydrogen Scattering Calculation. Phys. Rev. A 1974, 9, 219. [CrossRef]

8. Drachman, R.J. Positron Scattering Theory. In Physics of Electronic and Atomic Collisions, Proceedings of International Conference of the Physics of Electronic and Atomic Collisions VII, Amsterdam, The Netherlands, 26-30 July 1971; North-Holland Pub. Co.: Amsterdam, Netherlands.

9. Ghosh, A.S.; Sil, N.C.; Mandal, P. Positron-Atom and Positronium-Molecule Collisions. Phys. Rep. 1982, 87, 313. [CrossRef]

10. Bhatia, A.K. Positron Interactions with Atoms and Ions. J. At. Mol. Condens. Nano Phys. 2014, 1, 45.

11. Kadyrov, A.S.; Bray, I. Recent Progress in the Description of Positron Scattering from Atoms Using the Convergent Close-Coupling Theory. 2016. [CrossRef]

12. Armstead, R.L. Electron-Hydrogen Scattering Calculations. Phys. Rev. 1968, 171, 91. [CrossRef]

13. Ferrell, R.A. Theory of Positron Annihilations in Solids. Rev. Mod. Phys. 1956, 28, 308. [CrossRef]

14. Crannell, C.J.; Joyce, G.; Ramaty, R.; Werntz, C. Formation of the $0.511 \mathrm{MeV}$ Line in Solar Flares. Astrophys. J. 1976, 210, 582. [CrossRef]

15. Leventhal, M.; MacCallum, C.J.; Stang, P.D. Detection of $511 \mathrm{keV}$ Positron Annihilation Radiation from the Galactic Center Direction. Astrophys. Lett. 1978, 225, L11-L14. [CrossRef]

16. Cline, T.L. The unique cosmic event of March 5, 1979. Comment. Astrophys. 1980, 9, 13-21.

17. DiRienzi, J.; Drachman, R.J. Re-examination of a Simplified Model for Positronium-Helium Scattering. J. Phys. B 2003, 36, 2409.

18. Bhatia, A.K.; Drachman, R.J.; Temkin, A. Annihilation during Positron-Hydrogen Collisions. Phys. Rev. A 1974, 9, 223. [CrossRef]

19. Humberston, J.W. (London, England). Private communication, 1977.

20. Khan, A.; Ghosh, A.S. Positronium Formation in Positron-Hydrogen Scattering. Phys. Rev. B 1967, $123,129$. [CrossRef]

21. Bhatia, A.K. Hybrid Theory of $P$-wave Electron-Li ${ }^{2+}$ Elastic-scattering and Photoabsorption in Two-electron Systems. Phys. Rev. A 2013, 87, 042705. [CrossRef] 